\title{
Clinical versus Pathologic staging of Renal Tumors: Role of Multi-Detector CT Urography
}

Ahmed I Kamel ${ }^{1}$, Mohamed H. Badawy ${ }^{1}$, Hossam Elganzoury ${ }^{1}$, Amr Elkhouly ${ }^{1}$, Khalid Elesaily ${ }^{1}$, Eldahshan $\mathrm{S}^{1}$, Mohamed A. A. Ismail ${ }^{1}$, Mostafa F Elshafie ${ }^{2}$, Emam M Abdel Aziz ${ }^{3}$, Ahmed G El Baz ${ }^{1}$, Mamdouh A Roshdy ${ }^{1}$, Tarek R El Leithy ${ }^{1}$, Samir Ghobashy ${ }^{1}$, Ahmed M Kamal ${ }^{1}$

${ }^{1}$ Urology department, Theodor Bilharz Research Institute, Giza, Egypt

${ }^{2}$ Radiology department, Theodor Bilharz Research Institute, Giza, Egypt

${ }^{3}$ Radiodiagnosis department, Al Azhar University, Cairo, Egypt

Type of article: Original

\begin{abstract}
Introduction: Our ability to diagnose renal cell carcinoma (RCC) has increased in the past 30 years as a result of the extensive application of imaging techniques, such as ultrasonography, computed tomography, and magnetic resonance imaging. Multi-detector computed tomography (MDCT) remains the most appropriate imaging modality for the diagnosis and staging of RCC. The aim of this work was to compare the findings of MDCT with surgical pathology to determine the accuracy of delineating tumor size, localization, organ confinement, lymph node metastases, and the extent of tumor thrombus in the renal vein and inferior vena cava.

Methods: The clinical, surgical, and anatomo-pathologic records of 99 patients treated by nephrectomy (radical or partial) for solid renal tumors at Theodor Bilharz Research Institute and Nasser Institute from 2005 to 2011 were reviewed retrospectively. All cases were staged pre-operatively with abdominal MDCT (pre- and postcontrast enhancement) in addition to the routine biochemical, hematological, and radiological work-up. The tumors' histologic types were determined according to the WHO classification of renal tumors in adults in 2004, and staging was updated to the TNM 2010 system. Data were analyzed using the t-test.

Results: The mean age was 52 (range 21-73). Seventy-eight patients were males, and 21 patients were females (Male/Female ratio: 3.7:1). There were no significant differences in the mean tumor size between radiographic and pathologic assessments in different tumor stages. The overall incidence of lymph node invasion in surgical specimens was $76 \%$, whereas MDCT showed a positive incidence in $68.4 \%$ of cases (false negative result in 7 cases, $7.6 \%$ ).

Conclusion: Our findings indicated that MDCT urography is an accurate method to estimate renal tumor size, lymph node, vascular and visceral metastases preoperatively. Also, preoperative staging of renal tumors with MDCT represents a valuable and accurate tool.

Keywords: Multi-Detector CT Urography, renal cell carcinoma, staging
\end{abstract}

\section{Introduction}

The incidence of renal cell carcinoma (RCC) has been increasing, with RCC currently accounting for about $3 \%$ of all cancers worldwide, with the highest incidence in Western countries (1). It is by far the most common soft tissue malignant mass, and it accounts for $85 \%$ of all malignant masses of the kidney $(2,3)$. Its incidence has been increasing in the past 30 years as a result of wider application of imaging techniques, such as ultrasonography, computed tomography, and magnetic resonance imaging (1). The 2004 World Health Organization classification for renal neoplasms recognized several distinct histological subtypes of RCC. These subtypes include clear cell carcinoma (70\%), papillary RCC $(10 \%)$, chromophobe RCC $(5 \%)$, hereditary cancer syndrome $(5 \%)$, other rare tumors (such as multilocular cystic RCC, medullary carcinoma, collecting duct carcinoma, mucinous, spindle cell

\section{Corresponding author:}

Ahmed I Kamel, Urology department, Theodor Bilharz Research Institute, Giza, Egypt.

E-mail: Mazenmera66@gmail.com

Received: October 04, 2015, Accepted: November 20, 2015, Published: December 2015

iThenticate screening: November 05, 2015, English editing: December 02, 2015, Quality control: December 05, 2015

(C) 2015 The Authors. This is an open access article under the terms of the Creative Commons Attribution-NonCommercialNoDerivs License, which permits use and distribution in any medium, provided the original work is properly cited, the use is non-commercial and no modifications or adaptations are made. 
carcinoma, neuroblastoma-associated RCC and Xp 11.2 translocation- TFE3 carcinoma) and other unclassified lesions (4\%) (4). Multi-detector computed tomography (MDCT) remains the most appropriate imaging modality for the diagnosis and staging of RCC (5). Some investigators also have suggested that enhancement at MDCT may assist in the discrimination of clear cell RCC from oncocytoma, papillary RCC, and chromophobe RCC (6, 7). MDCT also can help in the assessment of invasions of the collecting system, since tumors separated or in contact with the collecting system on execratory phase CT images did not show collecting system invasion on histopathology (8). All these values of using MDCT for diagnosis and staging and helping to determine the subtypes of RCC will be useful in planning appropriate surgical treatment. In unresectable primary RCC, changes in selected CT parameters after two cycles of neoadjuvant Sunitinib might be associated with the potential for surgical resection (9). The standard treatment for patients with localized or advanced RCC is radical nephrectomy. However, in tumors that measure less than $4 \mathrm{~cm}$ and/or the mass is separated from sinus fat on the CT image, the invasion of muscular venous branch was decreased. This may help facilitate partial nephrectomy. The objective of this research was to compare the findings of MDCT i.e., clinical preoperative assessment with surgical pathology, i.e., postoperative pathological assessment, in order to determine how accurate it is in delineating tumor size, localization, organ confinement, lymph node metastases, and the extent of tumor thrombus in renal vein and inferior vena cava, thereby facilitating proper decision making in managing RCC cases.

\section{Material and Methods}

\subsection{Study design and population}

In this retrospective study, 99 patients who were treated by either radical or partial nephrectomy for solid renal tumours at Theodor Bilharz Research Institute and Nasser Institute were included in the study. These patients were managed during the period from November 2005 to December 2011, and their medical records were reviewed retrospectively. The study population was comprised of 78 male patients and 21 female patients (Male/Female ratio: 3.7:1) who ranged in age from 21 to 73 .

\subsection{Data collection}

The medical records of all patients included in the study were reviewed regarding full urological, clinical, and laboratory examination including: 1) history taking (for recent complaint, urinary stones, diabetes, hypertension, operations and drug allergy), 2) physical examination, and 3) laboratory tests, including complete blood count (CBC), bleeding profile, biochemical (kidney and liver function), and complete urine analysis.

\subsection{MDCT Technique}

All patients were staged pre-operatively with abdominal MDCT (pre- and post-contrast enhancement). MDCT examination of the urinary tract was done according to the following steps:

- Pre-contrast scan for whole urinary tract from kidney to bladder. This phase is important to assess precontrast attenuation of the enhancing masses.

- Injection of $100 \mathrm{ml}$ of non-ionic contrast media using automatic injector at injection rate of $4 \mathrm{ml} / \mathrm{sec}$.

- Arterial scan (corticomedullary): about 25 seconds after the start of intravenous contrast medium injection. This phase depicts the renal arterial anatomy, status of the renal veins, and early enhancement of the vascular lesions. Reconstructed maximum intensity projection (MIP) images in this phase revealed CT angiographic images.

- Nephrographic scan: at 80-120 seconds after the start of contrast medium injection. During this phase the kidneys are homogenously enhancing, which helps in differentiation between renal masses and normal parenchyma. This scan also includes the whole abdomen and pelvis to visualize the rest of the urinary tract and other organs.

- Excretory scan: at 4 - 5 minutes after the start of contrast medium injection. In some cases we used 250 cc of intravenous saline drip after the nephrographic scan to help in adequate ureteric opacification.

We used two MDCT machines, the first of which was SOMATOM Sensation 16, in the Cairo scan radiology center for 43 cases, and the second of which was the 4-section CT scanner (Toshiba Asteion Super4 at the Theodor Bilharz Research Institute) for seven cases. Scan parameters for SIEMENS Sensation 16 Slice CT Scanner are 16 x 0.75 $\mathrm{mm}$ detector collimation, $075-\mathrm{mm}$ slice thickness, $12 \mathrm{~mm}$ per rotation table speed, 5-mm reconstruction increment. In the Toshiba these parameters were $4 \times 2.5-\mathrm{mm}$ detector collimations, 2.5 slice thickness, 6 mm per rotation table speed, 3-mm reconstruction increment. Each examination of the urinary tract consisted of a large number of axial images in different phases. CT data from the multiple acquisitions were downloaded to an independent workstation 
to obtain thinner slices, and, then, multiplanar images (sagittal and coronal) were generated as well as 3D images, including maximum intensity projections and volume rendering images. Maximum intensity projections for arterial phase images give 3D images for the aorta and renal arteries similar to those of conventional angiography, while in delayed phase, they give 3D images for the collecting system, ureters, and bladder similar to those of the IVU (CT urography). The tumor's histologic type was determined according to the WHO classification of renal tumours in adults 2004 (3), and staging was updated to the TNM 2002 system (4). The student's t-test was used to compare the mean radiographic tumour size with the mean pathologic size. Values of $p<0.05$ were considered as the threshold for statistical significance.

\section{Results}

The mean age was 52 (range 21-73). There were 78 male patients and 21 female patients (ratio 3.7:1). Four patients were subjected to partial nephrectomy for angiomyolipoma, and three for oncocytoma. Ninety-two cases were diagnosed as RCC and were subjected to radical nephrectomy. Histological subtypes were clear cell type in 75 patients, chromophobe cell type in nine patients, papillary cell type in four patients and sarcomatoid in four patients. Clinical and pathological stages in patients who underwent radical nephrectomy are shown in Table 1 . Table 2 shows the mean radiographic and pathologic tumor size in the transaxial diameter. Tables 2 and 3 show that there were no significant differences in the mean tumor size between the radiographic and pathologic assessments in different tumor stages $(\mathrm{p}>0.05)$. The overall incidence of lymph node invasion in surgical specimens was $76 \%$, whereas MDCT showed a positive incidence in $68.4 \%$ of cases (false negative result in seven cases, $7.6 \%$ ). All cases of renal capsular invasion, suprarenal invasion, and renal vein thrombus were detected radiographically and confirmed pathologically.

Table 1. Clinical and pathological stages in patients who underwent radical nephrectomy

\begin{tabular}{|l|l|l|l|l|l|}
\hline Stage & T3b & T3a & T2 & T1b & T1a \\
\hline Clinical stage & 11 & 18 & 28 & 27 & 8 \\
\hline Pathological stage & 11 & 20 & 27 & 28 & 6 \\
\hline
\end{tabular}

Table 2. Mean radiographic and pathologic tumor size in the transaxial diameter

\begin{tabular}{|l|l|l|l|}
\hline Variable & Tumor size $(\mathrm{cm})$ & Mean $(\mathrm{SD})$ & $\mathrm{n}$ \\
\hline Radiographic & $2.1-15$ & $8.35(3.24)$ & 92 \\
\hline Pathologic & $2.0-15.4$ & $8.45(3.23)$ & 92 \\
\hline
\end{tabular}

Table 3. Mean tumor size measured radiographically and pathologically in different tumor sizes

\begin{tabular}{|l|l|l|l|}
\hline \multirow{2}{*}{ Variable } & \multicolumn{3}{|l|}{ Tumor size $(\mathrm{cm})$} \\
\cline { 2 - 4 } & $<4(\mathrm{n}=8)$ & $4-7(\mathrm{n}=27)$ & $>7(\mathrm{n}=8)$ \\
\hline Radiographic & $3.16 \pm 0.63$ & $5.59 \pm 0.88$ & $10.39 \pm 2.21$ \\
\hline Pathologic & $3.30 \pm 0.78$ & $5.73 \pm 0.86$ & $10.46 \pm 2.25$ \\
\hline
\end{tabular}

Data are expressed as mean \pm standard deviation

\section{Discussion}

The most common primary malignancy of the kidney is renal cell carcinoma (RCC), which accounts for $2-3 \%$ of all cancers (1-3). The extensive use of cross-sectional imaging modalities has resulted in the detection of more than $50 \%$ of RCCs (1-4). Multidetector computed tomography (MDCT) continues to be the most extensively available and effective modality for detecting renal cell carcinoma (RCC), and its staging accuracy can be as high as $91 \%$. With the improvement of the spatial resolution and ability of MDCT scanners to obtain multiplanar and 3Dreconstructions, the diagnostic performance of CT in characterizing RCC and in estimating the extent of the disease has been improved significantly. Important information can be obtained by CT examination and used to plan treatment. It also can be used to locate tumors and determine their size as well as to determine renal arterial and venous anatomy and their relationship to the pelvicaliceal system (7). When tumors are diagnosed in patients with RCC, the most important factors are the stage of the tumor, its nuclear grade according to Fuhrman, and its histologic subtype (1). The patient's prognosis and survival are affected significantly by stage of the tumor, so treatment must be planned and implemented with this in mind. The most commonly used method for determining the stage is the tumor, node, metastasis (TNM) classification system, and there is a close correlation between the 
stage and the potential for curing the disease $(1,9)$. The latest version of the TNM classification system was published in $2010(1,9)$.

The treatment of choice since 1969 has been radical nephrectomy with ipsilateral adrenalectomy (1). During the last few decades, there has been a growing trend for more limited surgical intervention, including adrenal-sparing radical nephrectomy, laparoscopic nephrectomy, and nephron-sparing partial nephrectomy (1-4, 10-13). Based on surgeon's expertise and skills, either an open, pure laparoscopic approach or a robot-assisted approach can be used to perform partial nephrectomy, and both nephron-sparing surgery (NSS) and radical nephrectomy (14, 15) reportedly have produced similar oncological outcomes. When it is feasible to use nephron-sparing techniques, the results of our work show that MDCT can be a safe tool for pre-operative assessment and decision making. This concept was supported by Athina and Maria 2015 (7), who stated that Multidetector multiphase CT with multiplanar and 3Ddisplays, with their high staging accuracy, are the primary imaging modalities for the detection of RCC. CT features may prove useful in differentiating RCC from benign tumors and help to characterize the histologic subtype of RCC before the surgery. Chen et al. studied 169 renal tumors treated with NSS and reported that CT overestimated the sizes of tumors to a greater extent than the histopathology report. But the size difference was only $0.22 \mathrm{~cm}$, which was of little clinical significance. This suggested that CT is an accurate method for measuring the sizes of renal tumors before surgery (16). If RCC were diagnosed at an early stage, physicians could plan more conservative treatment strategies. Computed tomography (CT) is considered to be the best approach for detecting staging of RCC. With the improvement of the spatial resolution of Multidetector CT (MDCT) and its ability to obtain multiphase imaging, further improvement in the diagnosis of RCC was made possible by multi-planar and three-dimensional reconstructions in any desired plane (17). MDCT's features can allow the differentiation of RCC from benign renal tumors (18).

\section{Conclusions}

The overall discrepancy was only $1 \mathrm{~mm}$ between the mean radiographic and pathologic sizes of tumors, and measurement errors did not seem to cause any significant stage shift. Our findings indicate that MDCT urography is an accurate method that can be used to estimate the sizes of renal tumors, lymph nodes, and vascular and visceral metastases before surgery. The evaluation of unenhanced CT scans and the enhanced corticomedullary and nephrogenic phases resulted in $92 \%$ sensitivity in the staging of RCC. Thus, the use of MDCT to stage renal tumors preoperatively makes it a very valuable and accurate tool.

\section{Acknowledgments:}

The authors sincerely thanks the Theodor Bilharz Research Institute and Nasser Institute for their help in this study.

\section{Conflict of Interest:}

There is no conflict of interest to be declared.

\section{Authors' contributions:}

All authors contributed to this project and article equally. All authors read and approved the final manuscript.

\section{References}

1) Zagoria RJ, Dyer RB, Wolfman NT, HinnGC, Chen YM. Radiology in the diagnosis and staging of renal cell carcinoma. Crit Rev Diagn Imaging. 1990; 31: 81-115. PMID: 2288659

2) Ljungberg B1, Campbell SC, Choi HY, Jacqmin D, Lee JE, Weikert S, et al. The epidemiology of renal cell carcinoma. Eur Urol. 2011; 60(4): 615-21. doi: 10.1016/j.eururo.2011.06.049. PMID: 21741761

3) Taccoen X1, Valeri A, Descotes JL, Morin V, Stindel E, Doucet L, et al. Renal cell carcinoma in adults 40 years old or less: young age is an independent prognostic factor for cancer-specific survival. Eur Urol. 2007; 51:980-87. doi: 10.1016/j.eururo.2006.10.025, PMID: 17092632

4) Verhoest G1, Veillard D, Guillé F, De La Taille A, Salomon L, Abbou CC, et al. Relationship between age at diagnosis and clinic pathologic features of renal cell carcinoma. Eur Urol. 2007; 51: 1298-1304. doi: 10.1016/j.eururo.2006.11.056, PMID: 17174023

5) Leslie JA, Prihoda T, Thompson IM. Serendipitous renal cell carcinoma in the post-CT era: continued evidence in improved outcomes. Urol Oncol. 2003; 21(1): 39-44. doi: 10.1016/S1078-1439(02)00205-3, PMID: 12684126 
6) $\mathrm{Hu}$ J, Mao Y, White K; Canadian Cancer Registries Epidemiology Research Group. Overweight and obesity in adults and risk of renal cell carcinoma in Canada. Soz Praventivmed. 2003 ; 48 :178-85. doi: 10.1007/s00038-003-2046-2, PMID: 12891869

7) Tsili AC1, Argyropoulou MI. Advances of multidetector computed tomography in the characterization and staging of renal cell carcinoma. World J Radiol. 2015; 7(6): 110-27, doi: 10.4329/wjr.v7.i6.110, PMID: 26120380, PMCID: PMC4473304

8) Ljungberg B, Bensalah K, Bex A, Canfield S, Dabestani S, Hofmann F, et al. European Association of Urology. Guidelines on renal cell carcinoma. 2014. Available from: http://uroweb.org/guideline/renal-cellcarcinoma/2

9) Ferlay J, Steliarova-Foucher E, Lortet-Tieulent J, Rosso S, Coebergh JW, Comber H, et al. Cancer incidence and mortality patterns in Europe: estimates for 40 countries in 2012. Eur J Cancer. 2013; 49: 1374-403, doi: 10.1016/j.ejca.2012.12.027, PMID: 23485231

10) American Cancer Society. Cancer facts and figures 2014. Available from: http://www.cancer.org/acs/groups/content/@research/documents/webcontent/acspc-042151.pdf

11) Sheth S, Scatarige JC, Horton KM, Corl FM, Fishman EK. Current concepts in the diagnosis and management of renal cell carcinoma: role of multidetector ct and three-dimensional CT. Radiographics. 2001; Spec No: S237-54, doi: 10.1148/radiographics.21.suppl_1.g01oc18s237, PMID: 11598260

12) Novick AC. Renal-sparing surgery for renal cell carcinoma. Ürol Clin North Am. 1993; 20: 277-82. doi: 10.1016/ j.juro.2011.02, PMID: 8493750.

13) Zincke H, Ghavamian R. Partial nephrectomy for renal cell cancer is here to stay--more data on this issue. J Urol. 1998; 159: 1161-2, doi: 10.1097/00005392-199804000- 00013, PMID: 9507822

14) MacLennan S, Imamura M, Lapitan MC, Omar MI, Lam TB, Hilvano-Cabungcal AM, et al. Systematic review of oncological outcomes following surgical management of localised renal cancer. Eur Urol. 2012; 61: 972-93, doi: 10.1016/j.eururo.2012.02.039, PMID: 22405593

15) Gratzke C, Seitz M, Bayrle F, Schlenker B, Bastian PJ, Haseke N, et al. Quality of life and perioperative outcomes after retroperitoneoscopic radical nephrectomy (RN), open RN and nephron-sparing surgery in patients with renal cell carcinoma. BJU Int. 2009; 104: 470-5, doi: 10.1111/j.1464-410X.2009.08439.x , PMID: 19239445.

16) Chen W, Wang L, Yang Q, Liu B, Sun Y. Comparison of radiographic and pathologic sizes of renal tumors. Int Braz J Urol. 2013; 39: 189-94, doi: 10.1590/S1677-5538.IBJU.2013.02.06, PMID: 23683665

17) Türkvatan A, Akdur PO, Altinel M, Olçer T, Turhan N, Cumhur T, et al. Preoperative staging of renal cell carcinoma with multidetector CT. Diagn Interv Radiol. 2009; 15: 22-30, PMID: 19263370.

18) Kim JI, Cho JY, Moon KC, Lee HJ, Kim SH. Segmental enhancement inversion at biphasic multidetector CT: characteristic finding of small renal oncocytoma. Radiology. 2009; 252: 441-8. doi: 10.2214/AJR.12.8616, PMID: 19508984. 